\title{
ROTATION SETS FOR HOMEOMORPHISMS AND HOMOLOGY
}

\author{
MARK POLLICOTT
}

\begin{abstract}
In this article we propose a definition of rotation sets for homeomorphisms of arbitrary compact manifolds. This approach is based on taking the suspended flow and using ideas of Schwartzmann on homology and winding cycles for flows. Our main application is to give a generalisation of a theorem of Llibre and MacKay for tori to the context of surfaces of higher genus.
\end{abstract}

\section{INTRODUCTION}

For homeomorphisms of the unit circle the rotation number is an important and much studied topological invariant. It is a single number which quantifies the long term dynamical behaviour of all orbits. There have been attempts to generalise this idea to other manifolds, most notably annuli and tori $[4,10]$. In these generalisations the rotation number is usually replaced by a more general "rotation set" which is contained in the line or the plane, respectively. Both the original definition (for the circle) and these generalisations (to annuli and tori) are based on lifting the homeomorphism to the appropriate universal covering space. (Intuitively, the rotation set corresponds to associating some form of "average displacement" to lifts of points on the covering space.)

In this note we shall consider an alternative definition of rotation sets which applies equally well to all homeomorphisms of compact manifolds, isotopic to the identity (but which reduces to the usual definitions for the archtypal examples of the circle, annulus and torus). We shall introduce the rotation set to quantify the "drift in homology" of orbits, following Schwartzmann's work on winding cycles [13].

Our viewpoint is motivated by the following simple example. Let $f: S^{1} \rightarrow S^{1}$ be an orientation preserving homeomorphism of the circle and assume that it has rotation number $\alpha$ (in the familiar sense). Let $V=S^{1} \times[0,1] /(x, 1) \sim$ $(f(x), 0)$ be the mapping torus and notice that $V \simeq S^{1} \times S^{1}$. Let $\tilde{f}_{t}: V \rightarrow$ $V$ be the associated suspension flow. Identifying points $x \in S^{1}$ with points $(x, 0) \in V$ the orbit $\left\{x, f(x), \ldots, f^{n}(x)\right\}$ for $f$ can be viewed as part of the orbit $\tilde{f}_{t}(x, 0)$ for time $0 \leq t \leq T$, say (where $\left.T=n\right)$. This curve in $V$ approximates the cycle $(\alpha T, T)$ in $H_{1}(V, \mathbb{R})=H_{1}\left(S^{1}, \mathbb{R}\right) \times \mathbb{R}$. After scaling by $T$ and removing the redundant second coordinate we can read off $\alpha \in H_{1}\left(S^{1}, \mathbb{R}\right)=\mathbb{R}$.

Received by the editors March 29, 1990.

1980 Mathematics Subject Classification (1985 Revision). Primary 58Fxx.

Key words and phrases. Rotation set, homology, winding cycles. 
In $\S 1$ we shall present a formal definition of the rotation set for quite general homeomorphisms and in $\S 2$ we shall give a more geometric interpretation and relate it to the more familiar definitions.

In $\S \S 3$ and 4 we shall present some results using this definition. As a general rule it seems that different (and more interesting) results occur for closed surfaces of higher genus rather than compact manifolds of higher dimension, with the torus representing the simplest example for both classes. (For example, of interest is a generalisation of a result of Llibre-MacKay [10] for tori to surfaces of higher genus (in $\S 3$ ). The proof uses Thurston's classification theorem for surfaces, for which the homology definition seems particularly well suited. However, the natural generalisation to manifolds of higher dimensions fails.) In the final section, $\S 5$ we describe some natural problems and related examples.

I am greatly indebted to William Parry for suggesting the connection with Schwartzmann's work and for several interesting discussions. I would also like to thank John Franks and Luisa Magalhães for their interesting comments, and the referee for his useful advice.

\section{Definitions}

Let $M$ be a compact connected manifold and let $f: M \rightarrow M$ be a homeomorphism (isotopic and identity). In this section we shall present a fairly formal definition of the rotation set which emphasizes the connection with Schwartzmann's work. In the next section we shall give an equivalent definition which is somewhat more intuitive and closer to the definitions in the usual cases of the circle, annulus and torus.

To give a framework in which to formulate our definition of rotation set we want to describe the first real cohomology and first real homology groups of the manifold. We can choose to define the cohomology of $M$ as follows.

The integer cohomology of $M$ is the additive abelian group $H^{1}(M, \mathbb{Z})$ given by isotopy classes of continuous maps $f: M \rightarrow S^{1}$ from $M$ into the unit circle i.e. $H^{1}(M, \mathbb{Z})=C^{0}\left(M, S^{1}\right) / \sim$ where $f \sim g$ denotes isotopy equivalence. We can then define the real cohomology of $M$ by $H^{1}(M, \mathbb{R})=H^{1}(M, \mathbb{Z}) \otimes_{\mathbb{Z}} \mathbb{R}$. (This corresponds to changing the coefficients from $\mathbb{Z}$ to $\mathbb{R}$.)

These definitions can be shown to be equivalent to the usual definitions of the integer and real first singular cohomology groups, for example, of the manifold $M$.

We define the mapping torus $V$ for $f: M \rightarrow M$ to be the compact connected manifold $V=M \times[0,1] /(x, 1) \sim(f x, 0)$. Since the homeomorphism $f$ is isotopic to the identity it is easy to see that $V$ is homeomorphic to $M \times S^{1}$.

There is a trivial relationship between the integer and real cohomologies of $M$ and those of its mapping torus $V$.

Lemma 1. $H^{1}(V, \mathbb{Z}) \cong H^{1}(M, \mathbb{Z}) \oplus \mathbb{Z}$ and $H^{1}(V, \mathbb{R}) \cong H^{1}(M, \mathbb{R}) \oplus \mathbb{R}$, where the isomorphisms are as additive groups.

Proof. Since we have observed that the above definitions correspond to the usual singular cohomologies it suffices to recall that since $V$ is homeomorphic to $M \times S^{1}$ then it follows from the familiar theory that $H^{1}(V, \mathbb{Z}) \cong$ $H^{1}(M, \mathbb{Z}) \oplus H^{1}\left(S^{1}, \mathbb{Z}\right) \cong H^{1}(M, \mathbb{Z}) \oplus \mathbb{Z}$ and similarly $H^{1}(V, \mathbb{R}) \cong H^{1}(M, \mathbb{R}) \oplus$ $H^{1}\left(S^{1}, \mathbb{R}\right) \cong H^{1}(M, \mathbb{R}) \oplus \mathbb{R}$.

We can denote the dual groups to the cohomology group $H^{1}(M, \mathbb{R})$ by 
$H_{1}(M, \mathbb{R})$ (i.e. $H^{1}(M, \mathbb{R})^{*}=H_{1}(M, \mathbb{R})$, where $H^{1}(M, \mathbb{R})^{*}$ represents linear functionals from $H^{1}(M, \mathbb{R})$, viewed as a real vector space into the real numbers). We call the additive group $H_{1}(M, \mathbb{R})$ the real homology of $M$. Similarly, the real homology $H_{1}(V, \mathbb{R})$ for $V$ is the dual group to $H^{1}(V, \mathbb{R})$.

Remark. We observed before that $H^{1}(M, \mathbb{R})$ corresponds to the first singular cohomology group for the manifold $M$ over the real numbers. The notation we adopted with $H_{1}(M, \mathbb{R})=H^{1}(M, \mathbb{R})^{*}$ is consistent with that for the first singular homology group, for example, for the manifold $M$ because of the Universal Coefficient Theorem.

Using the obvious linear extension we can observe the following lemma.

Lemma 2. $H^{1}(M, \mathbb{Z})^{*}=H_{1}(M, \mathbb{R})$ and $H^{1}(V, \mathbb{Z})^{*}=H_{1}(V, \mathbb{R})$, where the identification is by the natural bijection.

Proof. Clearly $H^{1}(M, \mathbb{Z}) \subset H^{1}(M, \mathbb{R})$ forms an integer lattice and any linear functional from $H^{1}(M, \mathbb{Z})$ into the reals defines a unique linear functional on $H^{1}(M, \mathbb{R})$.

We can use the homeomorphism $f: M \rightarrow M$ to define the suspended flow $\tilde{f}_{t}: V \rightarrow V$ by $\tilde{f}_{t}(x, u)=(x, u+t)$, with the appropriate identifications on $V$ (i.e. if $n \leq u+t \leq n+1$ then $\left.\tilde{f}_{t}(x, u)=\left(f^{n}(x), u+t-n\right)\right)$.

For any $v \in V$, and $T>0$ we can define a linear functional $\Lambda_{v, T} \in$ $C^{0}\left(V, S^{1}\right)^{*}$ by

$$
\Lambda_{v, T}(k)=\frac{1}{T} \int_{0}^{T} \frac{d}{d t} \arg [k]\left(\tilde{f}_{t} v\right) d t,
$$

where $k: V \rightarrow S^{1}$ is a continuous function with argument $\arg [k]$ (i.e. $k=$ $\left.e^{i \arg [k]}\right)$ where for the purposes of the above integral we choose the argument so as to be continuous along orbits. These linear functionals are describe by the following result of Schwartzmann.

Lemma 3. (i) For each $v \in V$ the family of linear functionals $\left\{\Lambda_{v, T}\right\}_{T \in \mathbb{R}^{+}} \subseteq$ $C^{0}\left(V, S^{1}\right)^{*}$ is equicontinuous in the weak ${ }^{*}$ topology.

(ii) For each $v \in V$ the limit points $\mathscr{F}_{v} \subseteq C^{0}\left(V, S^{1}\right)^{*}$ of this family are constant on $\sim$-equivalence classes (cf. [13]).

In particular, part (ii) of Lemma 3 tells us that each $\Lambda \in \mathscr{F}_{v}$ gives a welldefined element in $H^{1}(V, \mathbb{Z})^{*}$, and so, by using Lemma 2 , an element in $H_{1}(V, \mathbb{R})$. From Lemma 1 we know that $H^{1}(V, \mathbb{R}) \cong H^{1}(M, \mathbb{R}) \oplus \mathbb{R}$ or at the level of the dual spaces we can write $H_{1}(V, \mathbb{R}) \cong H_{1}(M, \mathbb{R}) \oplus \mathbb{R}$. Because of this decomposition we can write these linear functionals in terms of their components as $\Lambda_{v}=\left(\Lambda_{v}^{(1)}, V_{v}^{(2)}\right) \in \mathscr{F}_{v}$ where $\Lambda_{v}^{(1)} \in H_{1}(M, \mathbb{R}), \Lambda_{v}^{(2)} \in \mathbb{R}$ (since $\left.\mathbb{R}^{*}=\mathbb{R}\right)$.

Because of the explicit construction of the functionals $\left\{\Lambda_{T, v}\right\}$ and $\mathscr{F}_{v}$ we can immediately see that $\Lambda_{v}^{(2)} \equiv 1 \in \mathbb{R}$, i.e. the second component of these linear functionals is independent of all choices. Furthermore, if we write $v=$ $(x, u)$ then $\Lambda_{v}^{(2)}$ is independent of the value of $u$ and depends only on the first coordinate $x \in M$ (in fact, it depends only on the orbit of $x$ ).

Henceforth, we need only consider the components $\Lambda_{v}^{(2)} \in H_{1}(M, \mathbb{R})$ of the linear functionals $\Lambda_{v} \in \mathscr{F}_{v}$. We can denote these projections by $\mathscr{F}_{x}$, where 
$v=(x, u)$, say (in view of the above comments) and clearly there is a natural bijection $\mathscr{F}_{x} \rightarrow \mathscr{F}_{v}$ with $\Lambda_{x} \rightarrow \Lambda_{v}=\left(\Lambda_{x}, 1\right)$.

Definitions. We define the rotation set of $x \in M$ (relative to $f$ ) to be the subset of the real homology $\rho_{x}(f) \subseteq H_{1}(M, \mathbb{R})$ where $\rho_{x}(f)=\left\{\Lambda_{x} \mid \Lambda_{x} \in\right.$ $\left.\mathscr{F}_{x}\right\}$. We define the rotation set of $f$ to be the subset of the real homology $\rho(f) \subseteq H_{1}(M, \mathbb{R})$ such that $\rho(f)=\bigcup_{x \in M} \rho_{x}(f)$.

Remark. In fact the rotation set should be interpreted as being on the Albanese (or Jacobian) torus $H_{1}(M, \mathbb{R}) / H_{1}(M, \mathbb{Z})$. Henceforth we shall take this as understood and not refer to this point again.

We shall adopt the convention that if $\rho_{x}(f)$ consists of a single element then we call this the rotation vector (of $f: M \rightarrow M$ ).

The following easy observation will be useful later.

Lemma 4. If $f^{n} x=x$ is a periodic point then $\rho_{x}(f)$ is a single point in $H_{1}(M, \mathbb{R})$.

Proof. We can observe from the definitions that the families $\left\{\Lambda_{v, T}\right\}_{T \in \mathbb{R}^{+}}$are Cauchy in $T$ and thus convergent to a single point in $H_{1}(M, \mathbb{R})$.

Example 1.1. Let $M$ be a disc with two holes corresponding to two smaller disjoint discs removed from its interior (i.e. a "pair of pants" with three distinct boundary components). Since $M$ is contractible to a figure eight the $\mathbb{R}$-homology is given by $H_{1}(M, \mathbb{R})=\mathbb{R}^{2}$, with the obvious choice of generators. Given $\Lambda_{x} \in \mathscr{F}_{x} \subseteq H_{1}(M, \mathbb{R})$ the first coordinate measures the "average movement" of the orbit of $x$ around the first hole and the second coordinate the "average movement" of the orbit of $x$ around the second hole.

The three boundary components are homeomorphic to circles invariant under the homeomorphism $f$ and thus we can associate to the two inner boundary components rotation numbers $\rho_{1}, \rho_{2}$ and to the outer boundary component the rotation number $\rho_{3}$. For any $x \in \partial M$ we have rotation vectors $\left(\rho_{1}, 0\right)$, $\left(0, \rho_{2}\right)$ or $\left(\rho_{1}, \rho_{2}\right)$ depending on whether $x$ lies on one of the inner two boundary components or the outer component.

\section{GEOMETRIC INTERPRETATION}

In the previous section we gave a formal definition of the rotation set in terms of the homology of the manifold $M$. In this section we want to develop a better 'geometric' insight into the meaning of the rotation set and compare this definition with existing definitions in certain special cases.

Assume that $\Lambda \in \rho_{x}(f) \subseteq \rho(f) \subseteq H_{1}(M, \mathbb{R})$ is some element of the rotation set. By construction it corresponds to the second component of an element $\Lambda_{v}=\left(\Lambda_{v}^{(1)}, \Lambda_{v}^{(2)}\right) \in \mathscr{F}_{v}, v=(x, u)$, where $\mathscr{F}_{v}$ are the limit points of the family of linear functionals $\Lambda_{v, T} \in C^{0}\left(V, S^{1}\right)^{*}, T>0$, defined by

$$
\Lambda_{v, T}(k)=\frac{1}{T} \int_{0}^{T} \frac{d}{d t} \arg [k]\left(\tilde{f}_{t} v\right) d t .
$$

These linear functionals correspond to elements in the real homology of the mapping torus $V$. They can be approximated as follows (cf. [11]). Choose a convergent subsequence $\Lambda_{v}, T_{i} \rightarrow \Lambda_{v}, T_{i} \rightarrow+\infty$ and for each $T_{i}$ consider the orbit segment $\tilde{f}_{\left[0, T_{i}\right]}(v)$ contained in $V$. We can join up the two ends $v$ and 
$\tilde{f}_{T_{i}}(v)$ by an arbitrary curve of bounded length (the bound being determined by the diameter of the manifold) and denote the resulting closed curve in $V$ by $\gamma_{i}$. The closed curve $\gamma_{i}$ represents a cycle $\left[\gamma_{i}\right]$ in either the integer or real homology of $V$. Finally, we scale this cycle by $T_{i}$ to get an element $\left[\gamma_{i}\right] / T_{i}$ in the real homology $H_{1}(V, \mathbb{R})$ of $V$. The element $\Lambda \in H_{1}(V, \mathbb{R})$ is a limit point of the elements $\left[\gamma_{i}\right] / T_{i}$.

This gives a slightly more intuitive interpretation of the homological definition of rotation vector.

The homological definition of the rotation set is (essentially) consistant with the standard definition of rotation number for homeomorphisms of the circle using a lift of the homeomorphism to the real line. Furthermore, there also exist definitions of rotation sets for homeomorphisms of annuli and tori, again using lifts of the homeomorphism, for which the above definition of a rotation set reduces to the existing definitions in these special cases. [We should qualify this statement in two ways: firstly, we should recall these definitions give a rotation set modulo an appropriate integer lattice; and secondly, there are more than one alternative definition of the rotation set for tori in common use.]

For definiteness we shall concentrate on the special case of a homeomorphism $f: \mathbb{T} \rightarrow \mathbb{T}$ on the two-dimensional torus. The torus $\mathbb{T}$ has the two-dimensional plane $\mathbb{R}^{2}$ as its universal covering space and we can choose a lift $F: \mathbb{R}^{2} \rightarrow \mathbb{R}^{2}$ for the homeomorphism $f$. This lift will be defined up to translations by $\mathbb{Z}^{2}$. Given $(x, y) \in \mathbb{T}$ we choose a lift $(\tilde{x}, \tilde{y}) \in \mathbb{R}^{2}$ and consider the sequence

$$
\left\{\frac{F^{n}(\tilde{x}, \tilde{y})-(\tilde{x}, \tilde{y})}{n} \mid n \geq 1\right\} \subset \mathbb{R}^{2} .
$$

We define the geometric rotation set of $x \in M$ (relative to $f$ ) to be the subset of the plane $\rho_{x}^{g m}(f) \subseteq \mathbb{R}^{2}$ which are limit points of this sequence.

We define the geometric rotation set of $f$ to be the subset of the plane $\rho(f) \subseteq$ $\mathbb{R}^{2}$ such that $\rho^{g m}(f)=\bigcup_{x \in M} \rho_{x}^{g m}(f)$.

Remark. As in our previous definition of the rotation set the geometric rotation set should be understood as being defined up to an integer lattice (in this case $\left.\mathbb{Z}^{2}\right)$.

Proposition 1. For homeomorphisms of the torus we have $\rho^{g m}(f)=\rho(f)$.

Proof. For the torus $\mathbb{T}$ we know that the real homology corresponds to $H_{1}(\mathbb{T}, \mathbb{R})$ $=\mathbb{R}^{2}$ and the real homology of its mapping torus $V$ is $H_{1}(V, \mathbb{R})=\mathbb{R}^{3}$. By our previous comments the rotation set can be interpreted as limit points in the homology of elements constructed out of trajectories of the flow $\tilde{f}$ in $V$. In particular, $\rho_{x}(f), x \in M$, is the projection onto $H_{1}(M, \mathbb{R})$ of the limit points of $\left[\gamma_{i}\right] / T_{i} \in H_{1}(V, \mathbb{R})$, where $\gamma_{i}$ is some closed curve approximating a trajectory as described above.

We can lift the flow $\tilde{f}$ on $V$ to a flow $\widetilde{F}$ on the covering space $\widetilde{V}$ (observe that $\widetilde{V}$ is homeomorphic to $\left.\mathbb{R}^{2} \times \mathbb{R}\right)$. Let $(\tilde{x}, \tilde{y} ; 0) \in \widetilde{V}$ be a lift of $(x, y ; 0) \in V$ then it is easy to see that the displacements $F^{n}(\tilde{x}, \tilde{y})-(\tilde{x}, \tilde{y})$ are approximated by the element $\left(a_{i}, b_{i} ; n_{i}\right) \in \mathbb{Z}^{3}$ (in the covering group for $V$ ) which relates the endpoints of a lift $\tilde{\gamma}_{i}$ of the curve $\gamma_{i}$. Furthermore, because we are dealing with a torus we notice that $\left[\gamma_{i}\right]=\left(a_{i}, b_{i} ; n_{i}\right) \in H_{1}(V, \mathbb{R})=\mathbb{R}^{3}$. Observe that by construction the first coordinate (in $\left.\mathbb{R}^{2}\right)$ of $\widetilde{F}_{n}(\tilde{x}, \tilde{y})$ corresponds to $F^{n}(\tilde{x}, \tilde{y})$, for some appropriate choice of lift $F$. 

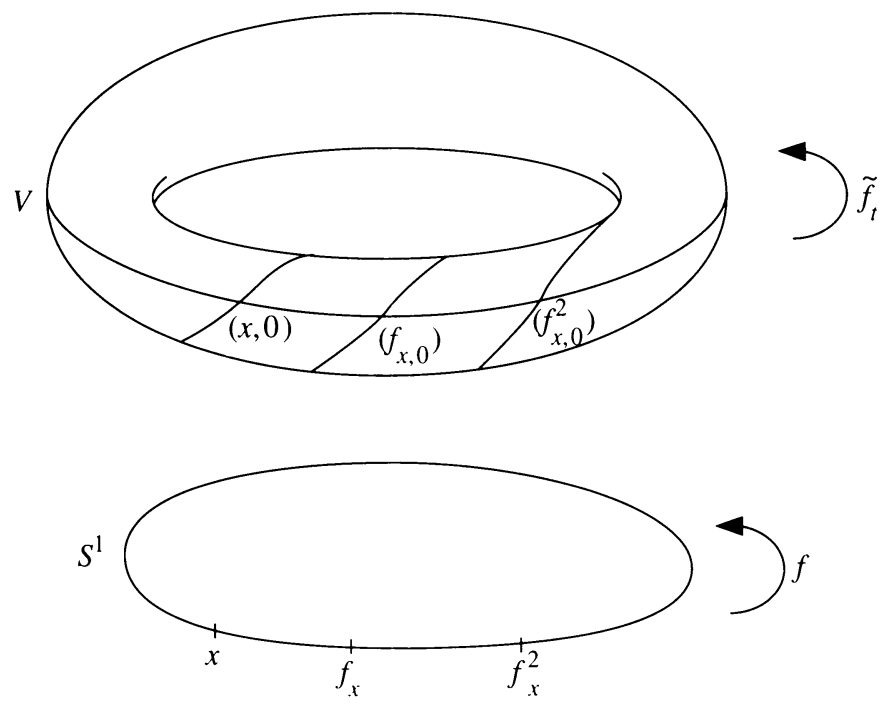

FIGURE 1

The projection $\left(a_{i}, b_{i}\right)$ onto $\mathbb{R}^{2}$ of the translation $\left(a_{i}, b_{i} ; n_{i}\right)$ associated to the lift $\tilde{\gamma}_{i}$ approximates the first coordinate of displacement $F^{n}(\tilde{x}, \tilde{y})$ to $(\tilde{x}, \tilde{y})$. It is now clear that the projection onto $H_{1}(M, \mathbb{R})$ of the limit points of $\left[\gamma_{i}\right] / T_{i}$ (or equivalently $\left(a_{i}, b_{i}\right) / T_{i} \in \mathbb{R}^{2}$ ) are coincident with the limit points of the sequence

$$
\left\{\frac{F^{n}(\tilde{x}, \tilde{y})-(\tilde{x}, \tilde{y})}{n} \mid n \geq 1\right\} \subset \mathbb{R}^{2} .
$$

This completes the proof. Similarly, the homology definition of rotation sets can be shown to be consistant with the geometric definitions for the circle, annulus and higher dimensional tori.

The following example nicely illustrates these different approaches.

Example 2.1. Let $M=S^{1}$ and $f: S^{1} \rightarrow S^{1}$ be given by $f(z)=z \cdot e^{2 \pi i c x}$, $\alpha \in \mathbb{R}$, i.e. a rotation through an angle $2 \pi \alpha$. The mapping torus $V$ is in fact the usual torus $V=\mathbb{R}^{2} / \mathbb{Z}^{2}$. The linear functionals $\Lambda_{v} \in H_{1}(V, \mathbb{R})$ reduce to the single functional $(\alpha, 1)$ and therefore $\Lambda_{x}=\alpha$, for every point $x$ on the circle. The number $\alpha$ is the rotation number in the usual sense. (See Figure 1.)

Example 2.2. Let $f: M \rightarrow M$ be a homeomorphism of a compact surface of genus $g \geq 2$. The surface $M$ has the open unit disc $D$ as its covering space and the fundamental group $\pi_{1}(M)$ is isomorphic to the covering group. Assume that $\Lambda \in \rho(f)$ is nonzero then it is approximated by the projection onto $H_{1}(M, \mathbb{R})$ of appropriately scaled orbit segments for the flow $\tilde{f}: V \rightarrow V$. Since these orbits segments are growing in homology the approximating curves $\gamma_{i}$ are growing in word length. The displacement of the end points of the projection onto $D$ of the lift $\tilde{\gamma}_{i}$ of this curve corresponds to an element of $\pi_{1}(M)$ i.e. an element of the covering group. 
However, in contrast to the case of the torus of genus one (which has abelian fundamental group) any geometric interpretation of the rotation set becomes very complicated precisely because we need to abelianise the fundamental group to get the homology.

All further references to rotation set will be relative to the above homologybased definition (with the comments in this section borne in mind).

\section{INVARIANT MEASURES}

In $\S 1$ we defined the rotation set via linear functionals. The Riesz representation theorem suggests that measure theory should play a useful role in applying this definition. In this section we shall develop this viewpoint.

Given $v \in V$ and $T>0$ we define a probability measure $\mu_{T, v}$ on $V$ by

$$
\int k d \mu_{T, v}=\frac{1}{T} \int_{0}^{T} k\left(\tilde{f}_{t} v\right) d t, \quad k \in C^{0}(V, \mathbb{R}) .
$$

Since the space of probability measures is weak* compact we can denote the limit points for a given $v \in V$, with $v=(x, u)$ by $\mathscr{M}_{x, f}$ (clearly the limit points depend only on $x$, and in fact only on the $f$-orbit of $x$ ). Such measures are easily seen to be $\tilde{f}_{t}$-invariant.

The relationship between the rotation set $\rho_{x}(f)$ and the family $\mathscr{M}_{x, f}$ is explained by the following result of Schwartzmann.

Proposition 2. $\rho_{x}(f)=\left\{\frac{1}{2 \pi i} \int\left(g^{\prime} / g\right)(w) d \mu(w) \mid \mu \in \mathscr{M}_{x, f}\right\}$, where $g \in C^{0}\left(V, S^{1}\right)$ and $g^{\prime}$ denotes differentiation in the flow direction (since $g$ can always be replaced by an element in the same equivalence class which is differentiable in this way).

This is essentially a reinterpretation of results from [13].

Let $\mathscr{M}_{f}$ denote the union of the measures $\mathscr{M}_{x, f}$ over all $x \in M$ i.e. $\mathscr{M}_{f}=$ $\bigcup_{x \in M} \mathscr{M}_{x, f}$.

Corresponding to Lemma 1 we have the following well-known result on measures.

Lemma 5. There is a bijection between $\tilde{f}$-invariant measures $\mu$ on $V$ and $f$ invariant measures $v$ on $M$, by $\mu=v \times l$, where $l$ denotes one-dimensional Lebesgue measure.

Thus we can also interpret $\mathscr{M}_{f}, \mathscr{M}_{x, f}$ as families of $f$-invariant measures on $M$ (by considering their projections on to the first coordinate using Lemma $5)$.

In view of Proposition 2 we can define a map $\mathscr{L}: \mathscr{M}_{f} \rightarrow \rho(f)$ with $\mathscr{L}\left(\mathscr{M}_{x, f}\right)$ $=\rho_{x}(f)$. We can now use well-known features of the space of invariant measures to prove less obvious results about the rotation set.

For any set $A$ in an affine vector space let $\overline{\operatorname{co}}(A)=\{\lambda x+(1-\lambda) y \mid x, y \in A\}$ denote its closed convex hull i.e. the smallest closed convex set containing $A$. For any convex set $C$ in an affine space let $\operatorname{Ext}(C)=\{z \in C \mid \exists x, y \in C$, $0 \leq \lambda \leq 1, z=\lambda x+(1-\lambda) y \Rightarrow \lambda=0,1\}$ be the extremal points of $C$.

Theorem 1. $\rho(f)$ contains the extremal points of its own closed convex hull i.e. $\rho(f) \supseteq \operatorname{Ext}[\overline{\operatorname{co}}(\rho(f))]$. 
Proof. Let $\mu$ be an $f$-invariant ergodic probability measure on $M$ and let $\mu \times l$ be the corresponding $\tilde{f}$-invariant ergodic probability measure on $V$. By the Birkhoff ergodic theorem applied to the flow $\tilde{f}$ it follows that there exists at least one point $v \in V$ (in fact, almost all points) such that $\mu_{v, T} \rightarrow \mu$, as $T \rightarrow+\infty$. Therefore we can conclude that $\mathscr{M}_{f}$ contains all ergodic measures for $\tilde{f}: V \rightarrow V$. By a well-known application of Choquet's theorem the ergodic measures are precisely $\operatorname{Ext}\left(\mathscr{I}_{f}\right)$, the extremal measures in the set of all $\tilde{f}$ invariant probability measures (where $\mathscr{I}_{f}$ is a closed convex set), thus we can write: (i) $\operatorname{Ext}\left(\mathscr{I}_{f}\right) \subset \mathscr{M}_{f}$; and (ii) $\overline{\operatorname{co}}\left(\mathscr{M}_{f}\right)=\mathscr{I}_{f}$.

Since $\mathscr{L}: \mathscr{M}_{f} \rightarrow \rho(f)$ is linear it has a natural extension $l: \mathscr{I}_{f} \rightarrow \overline{\mathrm{co}}(\rho(f))$ to the convex hulls of these sets (using (ii)). This extension is surjective since the map is linear and $\mathscr{L}\left(\mathscr{M}_{f}\right)=\rho(f)$ (using the comments after Lemma 5).

We claim that $\operatorname{Ext}\left(\mathscr{L}\left(\mathscr{I}_{f}\right)\right) \subset \mathscr{L}\left(\operatorname{Ext}\left(\mathscr{I}_{f}\right)\right)$. This is an easy exercise general for linear maps and convex sets. From (i) above we know that $\operatorname{Ext}\left(\mathscr{J}_{f}\right) \subset \mathscr{M}_{f}$ and therefore $\mathscr{L}\left(\operatorname{Ext}\left(\mathscr{I}_{f}\right)\right) \subset \mathscr{L}\left(\mathscr{M}_{f}\right)=\rho(f)$, and since $\mathscr{L}: \mathscr{I}_{f} \rightarrow \overline{\operatorname{co}}(\rho(f))$ is surjective we have $\mathscr{L}\left(\mathscr{J}_{f}\right)=\overline{\mathrm{co}}(\rho(f))$ putting together these three observations completes the proof.

Although we do not know if $\rho(f)$ must always be closed, we have the following:

Corollary 1.1. $\rho(f)$ cannot be an open set.

Corollary 1.2. Any extremal point of $\Lambda \in \rho(f)$ is the $\mathscr{L}$ image of some ergodic measure $\mu$. In particular, for almost all points $x \in M$ (with respect to $\mu$ ) we have $\rho_{x}(f)=\Lambda$.

Remarks. (i) Clearly, $\rho(f)=\bigcup_{x \in M} \rho_{x}(f)$ contains the smaller set $\rho^{\text {per }}(f)$ $=\bigcup_{f^{n} x=x} \rho_{x}(f)$. For generic $C^{1}$ diffeomorphisms we can see the following stronger version of the above result is true:

$$
\text { closure }\left[\rho^{\text {per }}(f)\right] \supseteq \operatorname{Ext}[\overline{\operatorname{co}}(\rho(f))] \text {. }
$$

To see this we recall Mañé's ergodic closing lemma which states that "for $C^{1}$ generic diffeomorphisms $f: M \rightarrow M$ those invariant probability measures supported on periodic $f$-orbits are dense in the ergodic invariant probability measures for $f$ " [11]. The above claim then follows in the same way as the previous theorem.

(ii) It is very easy to construct examples of homeomorphisms for which the rotation set is not convex. For example, consider the time-one map of a flow on the torus with parallel attracting and repelling closed orbits, which have different rotation numbers. The rotation set of the homeomorphism will consist of two distinct points in $\mathbb{R}^{2}$.

(iii) If we have a $C^{2}$ surface diffeomorphism $f: M \rightarrow M$ and $\Lambda$ is an extremal point corresponding to an ergodic measure of strictly positive entropy then we can find periodic points $x_{i}$ with rotation vectors $\Lambda_{i} \rightarrow \Lambda$. (This is a consequence of Katok's closing lemma for nonuniformly hyperbolic diffeomorphisms [9].)

We can develop this theme further and give other examples of results for $\rho(f)$ which follow from results for $f$-invariant measures (or equivalently, $\tilde{f}$ invariant measures, by Lemma 5) transferred by the linear map $\mathscr{L}$. 
We recall that the nonwandering set for a homeomorphism is defined to be the set $\Omega=\left\{x \in M \mid \forall\right.$ neighbourhoods $U$ of $x \exists n_{i} \rightarrow+\infty$ such that $\left.f^{n_{i}} U \cap U \neq \varnothing\right\}$. This is a compact $f$-invariant subset of $M$. We recall that any $f$-invariant measure must be supported on the nonwandering set $\Omega$ and so in particular any measure $\mu \in \mathscr{M}_{x, f}$ must be supported on $\Omega$. In view of Proposition 2 we see that in the definition of $\rho(f)$ it suffices to consider the union over $x \in \Omega$, rather than the whole manifold $M$. We summarise this as follows.

Proposition 3. $\rho(f)=\bigcup_{x \in \Omega} \rho_{x}(f)$.

Let $\mathscr{I}_{f}$ denote the family of $f$-invariant measures in the space $\mathscr{P}$ of all probability measures on $M$ (with the weak* topology). We recall that the map $f \rightarrow \mathscr{I}_{f} \subset \mathscr{P}$ is upper semicontinuous as a function of $f \in C^{0}(M, M)$ (cf. [12]) i.e. $\forall f \in C^{0}(M, M), \varepsilon>0 \exists$ neighbourhood $U \subset C^{0}(M, M)$ of $f$ such that $\forall g \in U, \mathscr{J}_{g} \subset B\left(\mathscr{J}_{f}, \varepsilon\right)$.

Proposition 4. $f \rightarrow \overline{\mathrm{co}}(\rho(f))$ is upper semicontinuous (i.e. $\forall f \in C^{0}(M, M)$, $\varepsilon>0 \exists$ neighbourhood $U$ of $f$ s.t. $\forall g \in U, \overline{\mathrm{co}}(\rho(g)) \subseteq B(\overline{\mathrm{co}}(\rho(f)), \varepsilon))$.

Proof. By Theorem 1 we know that $\rho(f)=\mathscr{L}\left(\mathscr{M}_{f}\right)$. Furthermore, by the above comments we see that the composition $f \rightarrow \mathscr{I}_{f} \stackrel{\mathscr{L}}{\rightarrow} \overline{\mathrm{co}}(\rho(f))$ is also upper semicontinuous.

\section{PERIODIC POINTS AND TOPOLOGICAL ENTROPY}

We shall concentrate in this section on the case where $M$ is a compact oriented surface of genus $g \geq 1$. For such surfaces we know that $H_{1}(M, \mathbb{R}) \cong \mathbb{R}^{2 g}$ (from singular homology theory).

We shall denote the topological entropy of the homeomorphism $f: M \rightarrow M$ by $h(f) \geq 0$. For tori (i.e. the case $g=1$ ) there is a result of MacKay-Llibre [10] (cf. also Franks [5]) which gives sufficient conditions for the homeomorphism $f: M \rightarrow M$ to have nonzero topological entropy $h(f)>0$ in terms of the rotation set and a finite number of periodic orbits.

In this section we shall present a generalisation of the MacKay-Llibre result to surfaces of higher genus using the homological definition of the rotation set. Our approach will be similar to that of MacKay-Llibre in that we shall depend heavily on the Thurston classification result for surfaces. (However, the further results of Franks for tori in [5] appear to use techniques which do not appear to have obvious generalisations to higher genus.)

The foundation of the proof is the Thurston Classification Theorem for isotopy classes of homeomorphisms of compact surfaces. We shall briefly summarize the results we need and refer the reader to [15, 3, and 2] for more information (and [8] for a related methodology).

Assume that $h: S \rightarrow S$ is a homeomorphism of a compact manifold (possibly with boundary). We call $h$ periodic if there exists $n>0$ such that $h^{n}$ is the identity on $S$. We call $h$ pseudo-Anosov if there exist certain $h$-invariant measurable foliations with a uniformly expanding transverse measure (cf. [15] for details). These two types of homeomorphism are distinct, and in particular periodic homeomorphisms have zero topological entropy and pseudo-Anosov 
homeomorphisms have nonzero topological entropy. (Furthermore, the pseudoAnosov homeomorphism minimizes the topological entropy within its isotopy class.)

Proposition 6 (Thurston Classification Theorem). Every homeomorphism $h: S$ $\rightarrow S$ is isotopic to a homeomorphism $k: S \rightarrow S$ such that:

(i) $k$ leaves invariant a finite family of disjoint simple closed curves $C_{1}, \ldots$, $C_{n}$ on $S$;

(ii) No curve $C_{i}$ is homotopic to a boundary curve of $S$;

(iii) We can decompose $S=\bigcup_{j=1}^{d} S_{j}$, where $S_{1}, \ldots, S_{d}$ are closed surfaces with disjoint interiors by cutting the surface $S$ along the curves $\left\{C_{1}, \ldots, C_{m}\right\}$.

(iv) For each $1 \leq j \leq d$, the homeomorphism $\left.k\right|_{S_{j}}: S_{j} \rightarrow S_{j}$ is either periodic or pseudo-Anosov.

We shall formulate our main result as follows:

Theorem 2. Let $f: M \rightarrow M$ be a homeomorphism of a compact closed surface of genus $g>1$. Assume that there exist $(2 g+1)$-periodic points $x_{1}, \ldots, x_{2 g+1}$ whose rotation vectors $\rho_{1}, \ldots, \rho_{2 g+1} \in H_{1}(M, \mathbb{R}) \equiv \mathbb{R}^{2 g}$ do not lie on a hyperplane then $h(f)>0$ and $\rho(f)$ is uncountable.

Proof. The strategy of the proof is to show that (some suitable modification of the homeomorphism is isotopic to a homeomorphism with a pseudo-Anosov component. The two results above (and many others) are natural corollaries of this fact.

By replacing $f$ by a suitable power we may assume that $\left\{x_{1}, \ldots, x_{2 g+1}\right\}$ are fixed points and $f$ is orientation preserving, and prove the result under these additional hypotheses. Let $V$ be the mapping torus of $f: M \rightarrow M$.

We can remove the fixed points $\left\{x_{1}, \ldots, x_{2 g+1}\right\} \subseteq M$ and then "blow-up" the missing points to (small) boundary circles $B_{1}, \ldots, B_{2 g+1}$. We shall denote the resulting surface (with these $2 g+1$ boundary components) by $S$.

Furthermore, we can replace $f: M \rightarrow M$ by a homeomorphism $h: S \rightarrow S$ with the same topological entropy, and dynamical features. (This is a standard construction, described in [1], for example.)

We can now apply the Thurston classification result to find a homeomorphism $k: S \rightarrow S$ homotopic to $h: S \rightarrow S$ which has the decomposition described in Proposition 6.

We begin by claiming that our hypothesis on fixed points implies that none of the decomposing curves $C_{i}$ can be contractible (viewed as simple closed curves on the original surface $M$ ). If any curve $C_{i}$ were contractible then it would correspond to the boundary of a contractible "disc" $D \subseteq M$ and $D \times[0,1] \subset V$ would be a tubular neighbourhood of the $\tilde{f}$-orbits of any of the fixed points contained in $D$. However, it is clear from the definition of a rotation set that if $D$ contained two or more fixed points then their $\tilde{f}$-orbits are homotopic in $V$. This implies that they must have the same rotation vector contradicting the hypothesis of the theorem. Alternatively, if $D$ contains a single fixed point it contradicts Proposition 6(ii). This proves our claim.

We next claim that the curves $C_{i}$ can only be separating curves for the original surface $M$ (i.e. curves which divide it into two disjoint pieces). If we assume that a curve $C_{i}$ is not separating, and we already know it is not contractible in $M$, then it must be homeomorphic to the "meridian" curve of a 
handle of $M$ [14]. In particular, we can choose the generators for the homology by taking a standard homology basis for $M$ and pulling it back under the above homeomorphism. Let $C_{i}^{*}$ denote the generator which intersects $C_{i}$. These observations show that $C_{i} \times[0,1] \subset V$ is an $\tilde{f}$-invariant submanifold which constrains the rotation vectors of the fixed points to lie on the hyperplane "orthogonal" to the generator $C_{i}$. This contradicts the hypotheses of the theorem and so proves the claim. We can also conclude that $d \leq g-1$.

We now know that each $C_{i}, i=1, \ldots, d$, must be a separating curve. We can thus divide the surface up into $(d+1)$ components. Furthermore, we can fill in the new boundary curves with discs to arrive at $(d+1)$ closed surfaces with genera $g_{1}, \ldots, g_{d+1}$, where $(d+1) \leq g_{1}+\cdots+g_{d+1}=g$. Assume that if the $i$ th such surface has $r_{i}$ of the original fixed points then $r_{1}+\cdots+r_{d+1}=2 g+1$. It follows that one of these surfaces must satisfy the hypotheses of the theorem and have no separating curves $C_{i}$. However, if this component corresponded to a periodic homeomorphism then the action on the homology corresponds to translation by a fixed vector in the integer lattice. In particular the periodic points could not have different rotation vectors. This contradiction proves that $k$ has a pseudo-Anosov component.

The implication that a pseudo-Anosov component implies $h(f)>0$ is now immediate from the Thurston theory (cf. [10, 15, 8], for example).

Remark. The two conclusions of the theorem are those that we considered the most interesting. However, the existence of a pseudo-Anosov component easily gives a variety of related results. For example:

(i) $\lim \sup _{n \rightarrow+\infty} \frac{1}{n}\left\{x \in M \mid f^{n} x=x\right\}>h(k)>0$ (cf. [15]).

This follows from Thurston's observation that the growth rate of periodic points is minimized within any isotopy class by the pseudo-Anosov homeomorphism (provided one exists) for which it equals the topological entropy. On those components for which no pseudo-Anosov homeomorphism exists in the isotopy class then the growth rate is subexponential, and so makes no contribution.

(ii) Let $x_{1}, \ldots, x_{r}$ be the periodic points lying in the component corresponding to the pseudo-Anosov homeomorphisms then: (a) $\overline{c o}\left\{x_{1}, \ldots, x_{r}\right\} \subseteq \rho(f)$; and for any closed set $F \subseteq \overline{c o}\left\{x_{1}, \ldots, x_{r}\right\}$ there exists $x \in M$ with $\rho_{x}(f)=F$ (cf. [10]). The proof rests on the fact that pseudo-Anosov homeomorphisms can be modelled using Markov partitions and symbolic dynamics, for which there are familiar tracing properties.

(iii) Let $\delta$ be a closed curve on $M$ which encloses at least two fixed points from $\left\{x_{1}, \ldots, x_{r}\right\}$ then $\frac{1}{n} \log \left|f_{*}^{n} \delta\right| \rightarrow h(k)>0$ where $|\delta|$ denotes the word length of $\delta$ as a curve in $\pi_{1}(S)$ (cf. [15]). This is a familiar property for pseudo-Anosov diffeomorphisms.

Example 4.1 (attributed to Michel Herman; cf. [10]). Let $M$ be the 3-torus and choose three disjoint cylinders parallel to each of the axes. We define $f: M \rightarrow M$ to be the identity exterior to the cylinders, translation by some rational number in the appropriate direction at the cylinder centers, and some continuous interpolation from the centre of the cylinders to their boundaries. It is easy to see that $h(f)=0$. However, the centers of the cylinders give rotation vectors (for periodic points) on the three axes of $H_{1}(M, \mathbb{R})=\mathbb{R}^{3}$ (with respect to the obvious basis for the homology). Furthermore, any point exterior to the cylinders is fixed with zero rotation vector. 


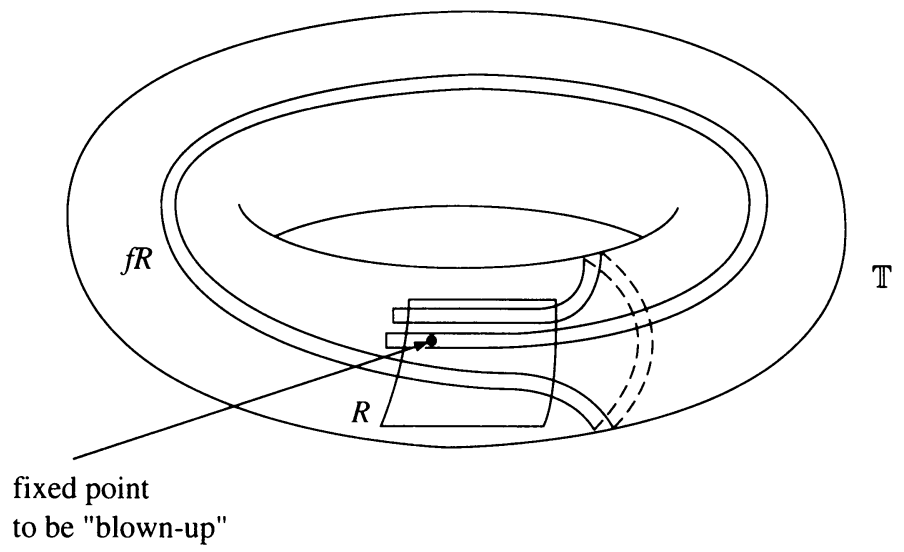

FIGURE 2

Example 4.2. Take two copies of the 2-torus and construct a homeomorphism with an invariant subset $\Lambda \subset \mathbb{T}$ conjugate to a full shift on two symbols (each state corresponding to each of the two natural generators for the homology). Take corresponding fixed point for each copy, and blow them up to a circle (in much the same way as in the proof of the theorem) and identify. This defines a homeomorphism on a surface of genus $g=2$, denoted $f: M \rightarrow M$. (See Figure 2.)

We can define a two dimensional simplex $\Delta \subseteq \mathbb{R}^{2}$ by $\Delta=\{(x, y) \mid x, y \geq 0$; $x+y \leq 1\}$. The homology of $M$ is again $H_{1}(M, \mathbb{R}) \equiv \mathbb{R}^{4}$ and relative to the standard homology basis $\Lambda$ corresponds to the sets $\Delta \times(0,0) \cup(0,0) \times \Delta \subseteq \rho(f)$.

Remark. In Example 2.1 we can easily construct a flow for which the time-one map $f: M \rightarrow M$ restricted to the boundary components has (rational) rotation numbers $\rho_{1}, \rho_{2}$ (for the inner circles) and $\rho_{3}$ (for the outer circle). Each boundary contains periodic points with these rotation numbers from the usual theory of diffeomorphisms of circles.

Recall that $H_{1}(M, \mathbb{R})=\mathbb{R}^{2}$, and the rotation vectors corresponding to boundary periodic points are $\left(\rho_{1}, 0\right),\left(0, \rho_{2}\right),\left(\rho_{3}, \rho_{3}\right) \in \mathbb{R}^{2}$. If we arrange that $\rho_{3} \neq\left(\rho_{1}+\rho_{2}\right) / 2$ then these points are not collinear. However, $h(f)=0$ since it is derived from a flow. (This shows the importance of assuming $M$ is a closed surface in the theorem.)

\section{EXAMPLES AND PROBLEMS}

In this section we shall give examples of rotation sets for various homeomorphisms, and pose what we consider to be natural questions. (As a general rule it is easier to construct counterexamples for manifolds of higher dimension than surfaces of higher genus.) Many more examples for the special cases of annuli and tori may be found in $[4-7,10,12]$.

Example 5.1. Let $M$ be a surface of genus 2 and let $f: M \rightarrow M$ be the homeomorphism (isotopic to the identity) giving rise to a modified "Smale horse-shoe" with hyperbolic behaviour on a rectangle $R$ as illustrated in Figure 3. The dynamics on $\Lambda=\bigcap_{n=-\infty}^{+\infty} f^{n} R$ corresponds to a subshift of finite type. 


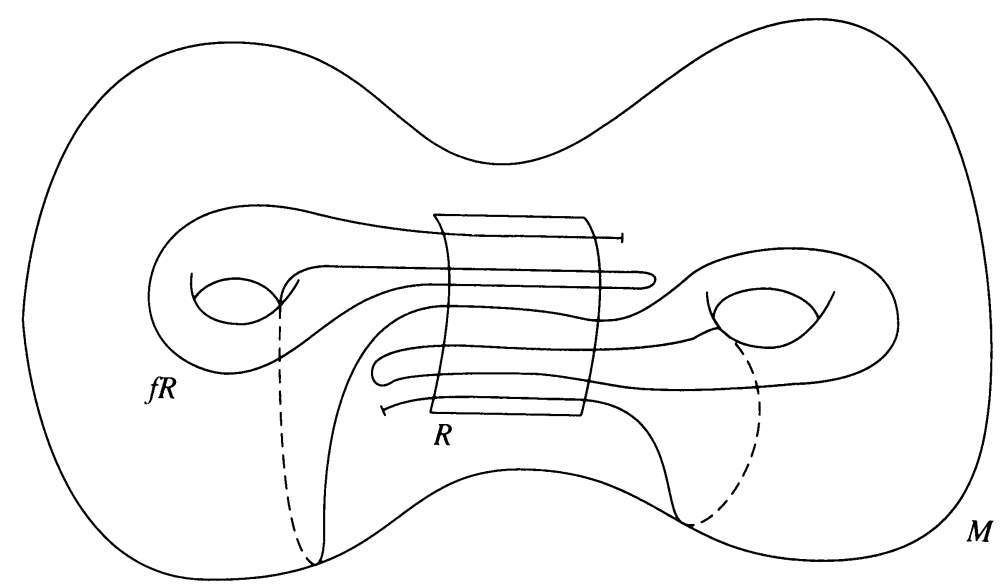

FIGURE 3

The homology of $M$ takes the form $H_{1}(M, \mathbb{R})=\mathbb{R}^{4}$, and (relative to the standard homology bases for $M)$ the union of the rotation sets of points in the set $\Lambda$ is a simplex $\Delta \subseteq H_{1}(M, \mathbb{R})$ of the form:

$$
\Delta=\{(x, y, z, w) \mid x, y, z, w>0 ; x+y+z+w \leq 1\} .
$$

We conclude with two problems which carry over from the case where $M$ is an annulus or a torus:

Problem 1. Is the set $\rho(f) \subseteq H_{1}(M, \mathbb{R})$ closed?

(For the special case of the annulus Mike Handel appears to have an affirmative answer, apparently using techniques connected with Thurston's theorem.)

Problem 2. If int $\rho(f) \neq \varnothing$ and $M$ is a surface then are the rotation vectors associated to periodic points dense to int $\rho(f)$ ?

(For tori John Franks has shown that this is indeed the case [5].)

Example 5.3. We give a counterexample to this problem in higher dimensions. Consider the standard Smale horse-shoe $h: S^{2} \rightarrow S^{2}$ and let $\Lambda$ denote the hyperbolic invariant set (corresponding to the full shift on two symbols) and let $p, q$ be the other two fixed points for $f$. We can write $\Lambda=\Lambda_{0} \cup \Lambda_{1}$ corresponding to the two symbols in the shift space. We can define a homeomorphism $f: M \rightarrow M$ on $M=S^{2} \times S^{1}$ such that $f$ is a skew product with

(i) $f_{1}=h$;

(ii) $\left.f_{2}\right|_{\Lambda_{0} \cup\{p, q\}}(z)=z \cdot e^{2 \pi i \alpha}$ and $\left.f_{2}\right|_{\Lambda_{1}}(z)=z \cdot e^{4 \pi i \alpha}$, where $\alpha$ is irrational. By construction $f: M \rightarrow M$ has no periodic points. Clearly, the first homology group satisfies $H_{1}(M, \mathbb{R})=\mathbb{R}$. Furthermore, it is easy to see that with the obvious generators the rotation set consists of the interval $[\alpha, 2 \alpha] \subset \mathbb{R}$.

Remark. The above example can be viewed as a time-one map for an approximate suspended flow over the Smale horseshoe map. In particular it shows that in general time-one flows need not satisfy the property that $\operatorname{int}[\rho(f)] \neq \varnothing$.

Problem 3. Find sufficient conditions for $\rho_{x}(f)$ to be a single vector for all $x \in M$. 


\section{REFERENCES}

1. R. Bowen, Entropy and the fundamental group, Lecture Notes in Math., vol. 668, Springer, 1978, pp. 21-29.

2. A. Casson and S. Bleiler, Automorphisms of surfaces after Nielsen and Thurston, Cambridge Univ. Press, Cambridge, 1988.

3. A. Fathi, F. Laudenbach, and V. Póenaru, Travaux de Thurston sur les surfaces, Astérique 66-67 (1979).

4. J. Franks, Generalisation of Poincaré-Birkhoff theorem, Ann. of Math. (2) 128 (1989), 139115.

5. __ Realising rotation vectors for torus homeomorphisms, Trans. Amer. Math. Soc. 331 (1989), 107-115.

6. __ Recurrence and fixed points of surface homeomorphisms, Ergodic Theory Dynamical Systems 8 (1988), 99-107.

7. J. Franks and M. Misiurewicz, Rotation sets of toral flows, preprint.

8. M. Handel, The entropy of orientation reversing homeomorphisms of surfaces, Topology 21 (1982), 291-296.

9. A. Katok, Lyapunov exponents, entropy and periodic orbits for diffeomorphisms, Inst. Hautes Études Sci. Publ. Math. 51 (1980), 137-173.

10. J. Llibre and R. MacKay, Rotation vectors and entropy for homeomorphisms of the torus isotopic to the identity, preprint.

11. R. Mañé, An ergodic closing lemma, Ann. of Math. (2) 116 (1982), 503-540.

12. M. Misiurewicz and K. Ziemann, Rotation sets for maps of tori, preprint.

13. S. Schwartzmann, Asymptotic cycles, Ann. of Math. (2) 66 (1957), 270-284.

14. J. Stillwell, Classical topology and combinatorial group theory, Springer, Berlin, 1980.

15. W. Thurston, On the geometry and dynamics of diffeomorphisms of surfaces, Bull. Amer. Math. Soc. 19 (1988), 417-431.

inic-Centro de Matematica, Faculdade de Ciencias, Universidade do Porto, 4000 Porto, Portugal 